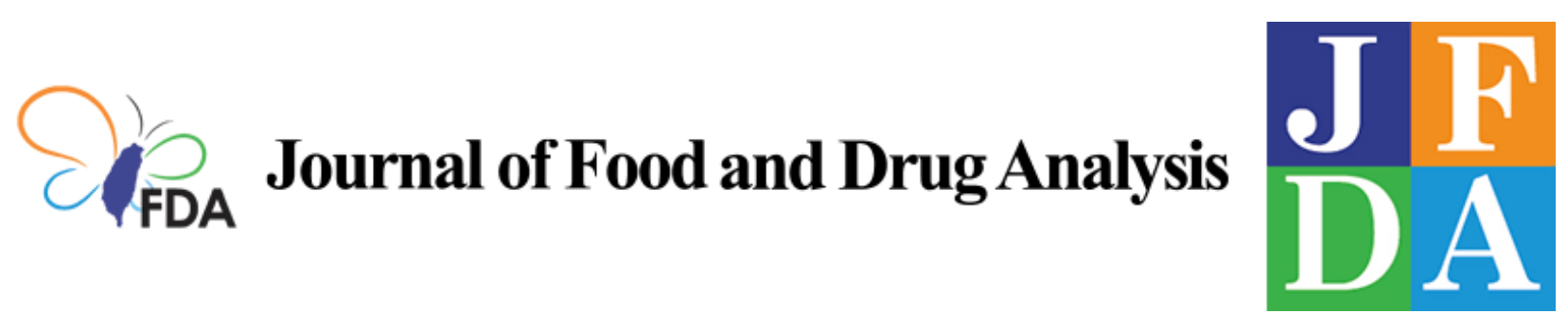

Volume 29 | Issue 3

Article 2

2021

\title{
Transcriptome analysis of Lactobacillus rhamnosus GG strain treated with prebiotic - bovine lactoferrin under a cold environment
}

Follow this and additional works at: https://www.jfda-online.com/journal

Part of the Food Science Commons, Medicinal Chemistry and Pharmaceutics Commons, Pharmacology Commons, and the Toxicology Commons

(c) (i) (9)

This work is licensed under a Creative Commons Attribution-Noncommercial-No Derivative Works 4.0 License.

\section{Recommended Citation}

Liu, Zhen-Shu; Lin, Chuen-Fu; and Chen, Po-Wen (2021) "Transcriptome analysis of Lactobacillus rhamnosus GG strain treated with prebiotic - bovine lactoferrin under a cold environment," Journal of Food and Drug Analysis: Vol. 29 : Iss. 3 , Article 2.

Available at: https://doi.org/10.38212/2224-6614.3369

This Original Article is brought to you for free and open access by Journal of Food and Drug Analysis. It has been accepted for inclusion in Journal of Food and Drug Analysis by an authorized editor of Journal of Food and Drug Analysis. 


\title{
Transcriptome analysis of Lactobacillus rhamnosus GG strain treated with prebiotic - bovine lactoferrin under a cold environment
}

\author{
Zhen-Shu Liu ${ }^{\mathrm{a}, \mathrm{b}}$, Chuen-Fu Lin ${ }^{\mathrm{c}}$, Po-Wen Chen ${ }^{\mathrm{d}, *}$ \\ ${ }^{a}$ Department of Safety, Health and Environmental Engineering, Ming Chi University of Technology, New Taipei City, 24301, Taiwan \\ ${ }^{\mathrm{b}}$ Chronic Diseases and Health Promotion Research Center, Chang Gung University of Science and Technology, Chiayi, 61363, Taiwan \\ ${ }^{c}$ Department of Veterinary Medicine, College of Veterinary Medicine, National Pingtung University of Science and Technology, \\ Pingtung, 91201, Taiwan \\ d Department of Veterinary Medicine, National Chung Hsing University, Taichung, 40249, Taiwan
}

\begin{abstract}
Lactoferrin (LF) is secreted by mammals and displays extensive biological effects. We previously reported that bovine LF (BLF) can boost the cold tolerance of a well-applied probiotic strain, Lactobacillus rhamnosus GG (LGG), to grow robustly under a cold environment, but the molecular mechanism is not clear. Here, RNA-seq analysis was conducted to ascertain molecular pathways underlying cold tolerance exerted by BLF. LGG was cultured in a cold environment $\left(22{ }^{\circ} \mathrm{C}\right)$ in the presence or absence of BLF. Transcriptome analysis indicated that BLF significantly elicited 1.2-3.2 fold (log2 Fold change) higher expression levels of genes related to stress, defense, cell division, and transporter in LGG, including the genes CspA, LytR, XRE, MerR, and GpsB. The KEGG pathway and GO analyses confirmed that BLF can modulate a few central pathways to boost the growth of LGG. BLF also reduced metabolic pathways involved in purine, amino acid, pyrimidine, one-carbon metabolism, and secondary metabolites in LGG. We speculate that the reduction of the above pathways may play key roles to reduce energy requirement and maintain carbon metabolism balance in LGG for surviving and growing in a cold state, and BLF can be an excellent prebiotic to LGG cultured in this cold condition (22 ${ }^{\circ} \mathrm{C}$ ). Overall, this study uncovers the molecular effects of BLF on LGG.
\end{abstract}

Keywords: Lactoferrin, Lactobacillus rhamnosus GG, Transcriptome

\section{Introduction}

$\mathrm{L}$ actoferrin (LF) is an $80-\mathrm{kDa}$ iron-binding protein present mostly in the milk and exocrine fluids of mammals, and it has been shown to display extensive biological effects including anti-inflammatory, antimicrobial, and immune-regulation properties. Thus, the application of LF to various fields has been studied [1-3]. For example, supplementation with LF or specific probiotic strains has been suggested to be a good strategy for controlling several bacterial and fungal vaginal infections, and a simultaneous combination of LF preparations and specific lactobacilli strains can improve women's health through probiotic and prebiotic input [4]. Although some studies have attempted to combine the health-promoting effects of LF use and specific probiotic bacterial strains together, LF may exert either growth inhibitory or promotion effects on specific probiotic strains [5-7]. Notably, LF exerts more favorable growth inhibitory effects on most pathogens than on probiotic strains, and the inhibitory effect of bovine LF (BLF) on selected probiotic bacteria is at least 4fold lower than that on several pathogenic bacteria $[6,8]$. In particular, we also revealed that at $22{ }^{\circ} \mathrm{C}$, the growth of Bifidobacterium breve,

Received 2 November 2020; revised 14 March 2021; accepted 17 June 2021.

Available online 15 September 2021.

* Corresponding author: No.145 Xingda Rd., South Dist., Taichung City, 40227, Taiwan.

E-mail address: powenchen@nchu.edu.tw (P.-W. Chen). 
Lactobacillus coryniformis, Lactobacillus delbrueckii, Lactobacillus acidophilus, Bifidobacterium angulatum, Bifidobacterium catenulatum, and Lactobacillus paraplantarum were completely blocked. However, these probiotics started regrowth in the presence of BLF $(1-32 \mathrm{mg} / \mathrm{mL})$ in a significant and dosedependent manner, supporting that LF possesses prebiotic activity on specific probiotic strains [7]. In other words, BLF supplementation can boost the stress tolerance responses of specific probiotic strains. However, the molecular mechanism of LF in boosting stress tolerance of specific probiotic strains is still unclear.

RNA sequencing (RNA-seq) and transcriptome analysis are good approaches for transcriptome profiling whereby deep-sequencing technologies are employed to directly determine the cDNA sequence. Moreover, this approach has a relatively low background compared with the use of microarrays. Most importantly, it provides the absolute expression value of each gene, thereby making it possible to evaluate the expression abundance of each gene in the same life stage $[9,10]$. Furthermore, RNA-seq can be applied to identify the transcriptional profiles of specific bacteria, and even strains or species whose genome have not yet been sequenced [9]. As for lactic acid bacteria, genes associated with the utilization of tetrasaccharides by Lactobacillus ruminis L5 were identified using transcriptome techniques [11], and the growth phaseassociated changes in the transcriptome profiles of Lactobacillus rhamnosus GG in industrial-type whey medium was also evaluated by RNA-seq [12]. Finally, RNA-seq has been conducted on Lactobacillus acetotolerans F28 growing in $12 \%$ ethanol to determine important genetic mechanisms for the short and long term adaption to ethanol stress [13]. Collectively, transcriptome analysis and RNA-seq can provide detailed information regarding global changes in gene expression and molecular pathways under particular conditions.

L. rhamnosus GG strain (ATCC 53103; LGG) was originally isolated from the human gastrointestinal tract as a probiotic bacterial strain $[14,15]$, and it has been shown to exert a variety of biological activities including antibacterial activity against a variety of bacterial species [16,17], immune modulation activities [18-20], and clinical trials also point out that this strain may reduce adiposity, body weight, and weight gain in human, and these suggest that LGG can be applied in the treatment of obesity [21]. Furthermore, several reports, including studies in different clinical conditions, human volunteer studies, and epidemiologic surveillance, all support that the LGG strain is safe for human consumption even in large amounts [22,23]. Collectively, LGG is currently one of the most widely studied, welldocumented, and safe probiotic bacterial strains; in our recent report, we demonstrated that BLF can strongly promote the growth rate of LGG and enhance the cold tolerance of LGG as well $[6,7]$. However, the mechanism of BLF becoming a prebiotic agent is still not well understood. In the current study, we employ transcriptome analysis to study the molecular mechanism of how BLF can strongly boost the growth of LGG grown under a cold environment. We identified evidence that BLF supplement can modulate a few central metabolic pathways in LGG and it also modulates specific stress-, defense-, cell cycle-, amino acid synthesisand transporter-related genes to encourage LGG adapt to the cold environment. This study investigates the role of BLF on the putative molecular regulatory network of LGG, and the identified differentially expressed genes (DEGs) in Kyoto Encyclopedia of Genes and Genomes (KEGG) termenriched terms may also help to secure core candidate genes for further functional studies on the molecular mechanism of stress tolerance in LGG. In the large scale fermentation of LGG that the combination of BLF and LGG could decrease the heat energy for LGG culture but also increase the output of the probiotics fermentation industry.

\section{Materials and methods}

\subsection{Bacterial strains, media, and growth conditions}

LGG ATCC 53103 was purchased from the Food Industry Research and Development Institute, Taiwan. Bacteria were first activated and maintained in MRS medium at $37{ }^{\circ} \mathrm{C}$ under anaerobic conditions for $48 \mathrm{~h}$. To evaluate the molecular mechanisms of BLF on the growth of LGG, a spectrophotometric turbidity bioassay was performed as described previously [7]. The broth of activated LGG (optical density was about 1.2) was diluted and adjusted to $5 \times 10^{6} \mathrm{cfu} / \mathrm{mL}$ and was further cultured at $22{ }^{\circ} \mathrm{C}$ in MRS broth medium supplemented with different concentrations of BLF when needed. Briefly, BLF was serially diluted in MRS broth, and $90 \mu \mathrm{L}$ of the prepared BLF was transferred into a 96-well culture plate containing $90 \mu \mathrm{L}$ of the prepared probiotic solution to achieve final LF concentrations of $32,16,8,4,2$, and $1 \mathrm{mg} /$ $\mathrm{mL}$. For the control cultures $(0 \mathrm{mg} / \mathrm{mL}$ of LF), MRS was added instead of the BLF solution. The growth responses of $\mathrm{LGG}$ at $22{ }^{\circ} \mathrm{C}$ in the presence or 
absence of BLF were measured by determining the optical density at $595 \mathrm{~nm}$ at different time intervals, and results are expressed as means $\pm \mathrm{SD}$. Moreover, at different time intervals, about $2 \mathrm{~mL}$ of bacterial cultures were harvested from 96-well plates and were further centrifuged at $6000 \times g$ for $10 \mathrm{~min}$ at $4{ }^{\circ} \mathrm{C}$ to collect the bacterial cells. Finally, the harvested bacterial cells were stored at $-80{ }^{\circ} \mathrm{C}$ and subjected to further RNA-seq analysis.

\subsection{RNA isolation and RNA-seq}

Total RNAs from four samples were extracted using TRIzol reagent (Invitrogen) and QIAGEN RNeasy mini kit. The total concentration, RIN, 23S/ 16S, and size of the RNA samples were determined using Agilent 2100 Bioanalyzer (Agilent RNA 6000 Nano Kit). The purity of the samples was evaluated by NanoDrop ${ }^{\mathrm{TM}}$. The total RNA was used to construct a cDNA library, and for transcriptome library construction, several workflows were completed, including rRNA depletion, RNA fragmentation, first strand cDNA synthesis, second strand cDNA synthesis, ends repair \& A-tailing, adaptor ligation, and PCR amplification. For example, DNase I digestion was used to degrade double-stranded and single-stranded DNA present in RNA samples. Then, total RNA samples were treated with Ribo-Zero ${ }^{\mathrm{TM}}$ Magnetic Kit to deplete rRNA, and RNA molecules were fragmented into small pieces using fragmentation reagent. As for cDNA synthesis, the first-strand cDNA was generated using random primers reverse transcription, and was followed by second-strand cDNA synthesis. Next, the synthesized cDNA was subjected to end-repair and then was $3^{\prime}$ adenylated. Finally, adapters were ligated to the ends of these 3' adenylated cDNA fragments.

As for PCR amplification, the cDNA fragments were amplified with adapters from the previous step, and PCR products were purified with the XP beads, and then dissolved in EB (RB solution). Next, the libraries were assessed for quality and quantity using two methods: first, the distribution of the fragment sizes was checked by Agilent 2100 bioanalyzer, and the library was quantified using realtime quantitative PCR (qPCR) (TaqMan Probe). Finally, qualified libraries were processed on cBot to generate the cluster on the flowcell, and the amplified flowcell was pair end sequenced on the Illumina System. Initially, according to the growth curve of LGG treated with or without the addition of $\mathrm{BLF}$, we focused on analyzing the transcriptome profiles of LGG co-cultured with $1 \mathrm{mg} / \mathrm{mL}$ BLF at $60 \mathrm{~h}$ and $72 \mathrm{~h}$ time intervals.

\subsection{Transcriptomics analysis}

A series of quality control steps were conducted on the raw reads obtained by sequencing. For example, we removed reads containing adaptor sequence, reads with an unknown base number greater than $5 \%$ of the total sequence length, reads with $\mathrm{Q} \leq 15$, and reads where the base number accounts for more than $20 \%$ of the total read base number of the reads. Then, the clean reads for each sample were aligned to the reference genome using HISAT software (v2.0.1-beta) [24].

DEGs were identified as described previously using PossionDis software [25], and the $\log 2$ (fold change) $\geq 1$ and false discovery rate $\leq 0.005$ were set as the significant threshold values to $S$ using the KEGG pathway database [26], and $Q$ value $\leq 0.05$ was set to ascertain the significantly enriched pathways. Moreover, GO enrichment analysis was conducted to determine the main molecular functions, cellular components, and biological processes associated with DEGs by consulting the to GO database (http://www.geneontology.org), and the corrected $p$-value $\leq 0.05$ was set to ascertain significantly enrichment GO terms. The cluster analysis for genome-wide expression with open source clustering software was determined as described previously $[27,28]$.

\section{Results}

\subsection{Influence of BLF on the growth of LGG}

To investigate the transcriptome profile of LGG in the presence or absence of BLF addition, LGG was cultured under a cold environment with a series of BLF concentrations (from 0 to $32 \mathrm{mg} / \mathrm{mL}$ ). As expected, in the absence of BLF treatment (Fig. 1), the growth of LGG was strongly retarded by lower temperature incubation up until $60 \mathrm{~h}$. LGG started slow regrowth at $72 \mathrm{~h}$, whereby the optical density (OD) of LGG elevated from below $0.2(60 \mathrm{~h})$ to about $0.5(72 \mathrm{~h})$ in $600 \mathrm{~nm}$. The regrowth of LGG at $72 \mathrm{~h}$ may be due to the adaption of bacteria to suboptimal growth conditions. In contrast, in the presence of BLF (1-32 $\mathrm{mg} / \mathrm{mL})$, LGG started regrowth in a significant and dose-dependent manner at $48 \mathrm{~h}$, $60 \mathrm{~h}$, and $72 \mathrm{~h}$ time points. Most importantly, the growth of LGG treated with either concentrations of BLF was observed to be at least 2-fold higher than the control at $60 \mathrm{~h}$ and $72 \mathrm{~h}$ time intervals. Notably, $1 \mathrm{mg} / \mathrm{mL}$ BLF was enough to display strong prebiotic activity towards LGG especially at $60 \mathrm{~h}$ and $72 \mathrm{~h}$ time points when compared to the control $(0 \mathrm{mg} /$ $\mathrm{mL}$ ). Thus, the transcriptome profiles of LGG 


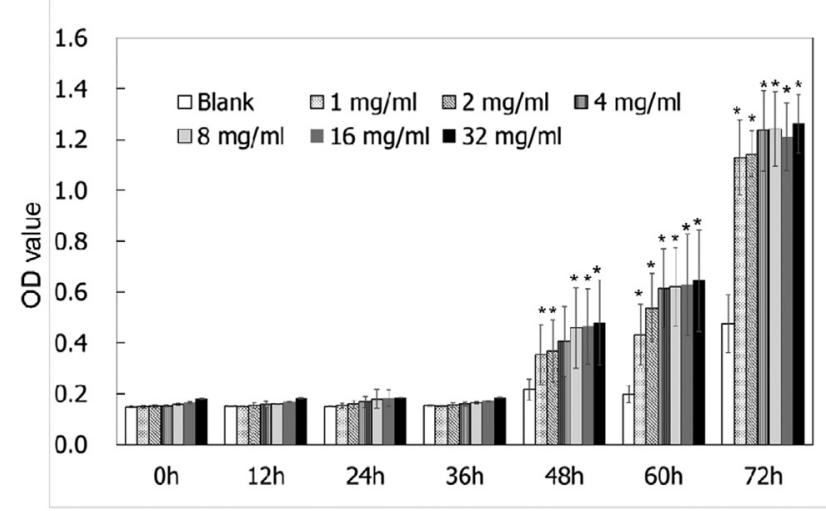

Fig. 1. Effects of bovine lactoferrin (BLF) on the growth of probiotics. Lactobacillus rhamnose GG strain (LGG) was cultured in MRS medium with and without various concentrations of BLF at $22{ }^{\circ} \mathrm{C}$. The growth responses of LGG were measured by determining the optical density. *Significant differences in the probiotic growth with and without BLF $(p<0.05)$.

treated with $1 \mathrm{mg} / \mathrm{mL}$ BLF at $60 \mathrm{~h}$ and $72 \mathrm{~h}$ were subsequently investigated. For comparison, the transcriptome profiles of LGG in the absence of BLF at $60 \mathrm{~h}$ and $72 \mathrm{~h}$ time points were also determined.

\subsection{The transcriptome of $L G G$ in the presence or absence of $B L F$}

In Table 1, sequence statistics of LGG are shown. We detected a total of 7.9 million pairs of reads for the control LGG at $60 \mathrm{~h}, 8.0$ million for the BLFtreated LGG at $60 \mathrm{~h}, 7.0$ million for the control LGG at $72 \mathrm{~h}$, and 7.3 million for the BLF-treated LGG at 72 h. Moreover, saturation curves and gene coverage indicated a completely adequate sequencing depth (Fig. S1 and S2). When compared to the known mRNA, the gene expression numbers in each sampler or the mean RPKM (Reads Per Kilo bases per Million reads) was 2358 in the control LGG at $60 \mathrm{~h}, 2371$ in the BLF-treated LGG at $60 \mathrm{~h}$, 2312 in the control LGG at $72 \mathrm{~h}$, and 2304 in the BLFtreated LGG at $72 \mathrm{~h}$ (Fig. S3). Moreover, high and low gene expressions are usually defined by an arbitrary threshold, and here, we defined genes with

Table 1. RNA-sequence statistics of LGG. LGG were cultured at $22^{\circ} \mathrm{C}$ in the presence or absence of BLF incubation from 0 to $72 \mathrm{~h}$. Control $(0 \mathrm{mg} /$ $\mathrm{mL})$ and BLF-treated $(1 \mathrm{mg} / \mathrm{mL})$ bacterial samples were analyzed using transcriptome analysis at $60 \mathrm{~h}$ and $72 \mathrm{~h}$ time interval.

\begin{tabular}{|c|c|c|c|c|}
\hline & $\begin{array}{l}\text { Control } \\
(60 \mathrm{~h})\end{array}$ & $\begin{array}{l}\text { BLF treated } \\
(60 \mathrm{~h})\end{array}$ & $\begin{array}{l}\text { Control } \\
(72 \mathrm{~h})\end{array}$ & $\begin{array}{l}\text { BLF treated } \\
(72 \mathrm{~h})\end{array}$ \\
\hline Total CleanReads & $7,926,940$ & $8,047,484$ & $7,048,228$ & $7,338,506$ \\
\hline $\begin{array}{l}\text { Total Mapped } \\
\text { Reads }(\%)\end{array}$ & 58.87 & 62.23 & 64.82 & 65.31 \\
\hline Perfect Match (\%) & 21.13 & 21.31 & 24.31 & 24.81 \\
\hline Unique Match (\%) & 57.64 & 61.44 & 64.52 & 64.97 \\
\hline
\end{tabular}

RPKM smaller than 10 as low expression genes, genes with RPKM levels between 10 and 100 as intermediate expression, and genes with RPKM levels above 100 as high. In Fig. S4, the boxplots of gene expression numbers of each sample indicate that the four samples showed similar gene expression levels. Furthermore, the hierarchical clustering of gene expression (Fig. S5) indicate that BLF treatment affects the gene expression pattern, and the most differentially expressed genes between control and BLF-treated LGG had relatively low to medium expression levels. These up-regulated and downregulated DEGs were further analyzed using PossionDis software as described below.

\subsection{Identification of DEGs}

To identify genes involved in prebiotic effects of BLF, we used PossionDis software to determine the DEGs between the samples. At the $60 \mathrm{~h}$ time point (Fig. S6), transcriptome analysis identified a total of 115 DEGs in BLF-treated LGG, including 59 upregulated genes and 56 down-regulated genes, and a total of 2293 genes were significantly non-regulated. At the $72 \mathrm{~h}$ time interval (Fig. S6), transcriptome analysis revealed that only 1 gene was up-regulated and 6 genes were down-regulated in BLF-treated LGG, and a total of 2327 genes were significantly non-regulated. In addition, we further extracted and summarized some DEGs that are useful for illustrating the molecular mechanism of BLF on promoting LGG as indicated below.

\subsection{Stress and defense response in LGG treated with BLF supplementation}

As described above, a total of 115 DEGs were identified in BLF-treated LGG at $60 \mathrm{~h}$ using PossionDis analysis. In the current study, LGG were cultured in a cold environment $\left(22^{\circ} \mathrm{C}\right)$, and thus, we speculate that the expression of genes involved in cell division or stress responses would be modulated by BLF supplementation. Indeed, among the 115 DEGs, expression of specific genes involved in stress or defense response pathway were promoted by BLF supplementation (Table 2). Here, about ten genes involved in stress, defense, or cell division responses were increased by $1.0-3.2$ fold ( $\log 2$ Fold change) in BLF-treated LGG, and the data of $p$-value and False discovery rate (FDR, $q$-value) have been shown as well. The FDR values for above genes all are below 0.004 (between 0.004 to smaller than 0.00001 ), and this supports that these genes are differently expressed in a considerately significant manner. For example, expression of $\mathrm{ABC}$ transporter permease (MFS 
Table 2. The fold change value of genes, grouped into stress, defense and cell division in LGG treated with and without BLF incubation at $60 \mathrm{~h}$ time interval. LGG were cultured at $22^{\circ} \mathrm{C}$ in the presence or absence of BLF incubation from 0 to $72 \mathrm{~h}$. Gene expression levels of control (0 mg/mL) and BLF-treated $(1 \mathrm{mg} / \mathrm{mL})$ bacterial samples were analyzed using RNA-seq analysis at $60 \mathrm{~h}$ and $72 \mathrm{~h}$ time interval. A total of 59 genes were up-regulated in BLF-treated LGG at $60 \mathrm{~h}$ time interval, and genes which are related to stress, defense and cell division genes are further summarized here. The $p$ value and FDR (False discovery rate, q-values) $<0.05$ indicate these genes are differently expressed in a statistically significant manner.

\begin{tabular}{|c|c|c|c|c|c|}
\hline Gene ID & $\begin{array}{l}\text { Log2 Fold Change } \\
\text { (treated/control) }\end{array}$ & Protein encoded & Functional group & FDR & p-value \\
\hline LRHM_RS11665 & 3.22 & $\mathrm{ABC}$ transporter permease & Stress response & 0.00035 & 0.00009 \\
\hline LRHM_RS02895 & 2.20 & Cold-shock protein (beta-ribbon, CspA family) & Defense mechanism & 0.00000 & 0.00000 \\
\hline LRHM_RS12300 & 2.08 & PTS galactitol transporter subunit IIA & Defense mechanism & 0.00000 & 0.00000 \\
\hline LRHM_RS10720 & 1.77 & LytR family transcriptional regulator & Defense mechanism & 0.00000 & 0.00000 \\
\hline LRHM_RS02995 & 1.49 & XRE family transcriptional regulator & Defense mechanism & 0.00100 & 0.00028 \\
\hline LRHM_RS11355 & 1.33 & XRE family transcriptional regulator & Defense mechanism & 0.00049 & 0.00013 \\
\hline LRHM_RS02560 & 1.18 & MerR family transcriptional regulator & Stress response & 0.00133 & 0.00038 \\
\hline LRHM_RS13960 & 1.14 & Peptide ABC transporter ATP-binding protein & Stress response & 0.00468 & 0.00154 \\
\hline LRHM_RS07940 & 1.06 & Peptide ABC transporter ATPase & Stress response & 0.00000 & 0.00000 \\
\hline LRHM_RS10305 & 1.01 & $\begin{array}{l}\text { Glycine/betaine } \mathrm{ABC} \text { transporter } \\
\text { ATP-binding protein }\end{array}$ & Stress response & 0.00007 & 0.00002 \\
\hline LRHM_RS07080 & 1.21 & Cell division protein & $\begin{array}{l}\text { Cell division and } \\
\text { cell cycle }\end{array}$ & 0.00000 & 0.00000 \\
\hline
\end{tabular}

transporter) and Cold-shock protein (CspA family) were enhanced by 3.2 and 2.2 fold, respectively. Moreover, genes encoding LytR and MerR family transcriptional regulator were up-regulated by 1.77 fold and 1.18 fold, respectively. Finally, a gene encoding MerR family transcriptional regulator (activator of the bmr gene), which can promote transcription of various stress regulons, was also upregulated by 1.18 fold. Collectively, these findings indicated that BLF supplementation could protect LGG against cold stress by up-regulating a series of stress- and defense-related genes especially at $60 \mathrm{~h}$. Notably, a cell division protein, GpsB, was also upregulated by 1.21 fold (log2 Fold change). In contrast, at $72 \mathrm{~h}$, there were only seven DEGs that were modulated by BLF supplementation, of which there were no stress- or defense-related genes that were up-regulated by BLF supplementation.

\subsection{Metabolic pathways and gene ontology (GO) analysis of DEGs}

KEGG pathway and GO analyses were conducted to interpret the potential roles played by the DEGs that promote LGG growth exerted by BLF. At $60 \mathrm{~h}$ (Fig. 2A), the identified significantly DEGs were mapped into four categories of pathways: cellular processes (3 genes), environmental information processing ( 7 genes), genetic information processing (7 genes), and metabolism (53 genes). In contrast, at $72 \mathrm{~h}$ (Fig. 2B), the identified significantly DEGs were mapped to only the metabolism category (8 genes). Collectively, there were more categories of pathways that were modulated by BLF at $60 \mathrm{~h}$ but not at $72 \mathrm{~h}$.
In addition, the top 20 enriched KEGG pathways are further shown in Fig. 3. Here, at $60 \mathrm{~h}$ (Fig. 3A), genes involved in "alanine, aspartate and glutamate metabolism", "one carbon pool by folate", "metabolic pathways", "biosynthesis of secondary metabolites", "biosynthesis of antibiotics", "purine metabolism", and "pyrimidine metabolism" categories were the most significantly enriched. On the other hand, at $72 \mathrm{~h}$ (Fig. 3B), genes involved in "alanine, aspartate and glutamate metabolism", "one carbon pool by folate", "metabolic pathways", "biosynthesis of secondary metabolites", "biosynthesis of antibiotics", and "purine metabolism" categories were the most significantly enriched. Therefore, the above findings from samples collected at $60 \mathrm{~h}$ and $72 \mathrm{~h}$ confirmed that BLF exerted predominantly on similar (metabolic pathways) as well as specific pathways to promote the growth of LGG.

We further compared the DEGs that were downregulated or up-regulated by BLF supplementation. At the $60 \mathrm{~h}$ time point (Fig. 4A), the most enriched pathways in BLF-treated contained down-regulated DEGs. For example, some genes involved in purine metabolism, alanine, aspartate, and glutamate metabolism, pyrimidine metabolism, biosynthesis of secondary metabolites, one carbon pool by folate, and biosynthesis of antibiotics were identified to be down-regulated by BLF supplementation. Moreover, genes of some pathways were also up-regulated, including RNA degradation, nicotinate and nicotinamide metabolism, butanoate metabolism, cell cycle-Caulobacter, base excision repair, DNA replication, peptidoglycan biosynthesis, aminoacyltRNA biosynthesis, cysteine and methionine 

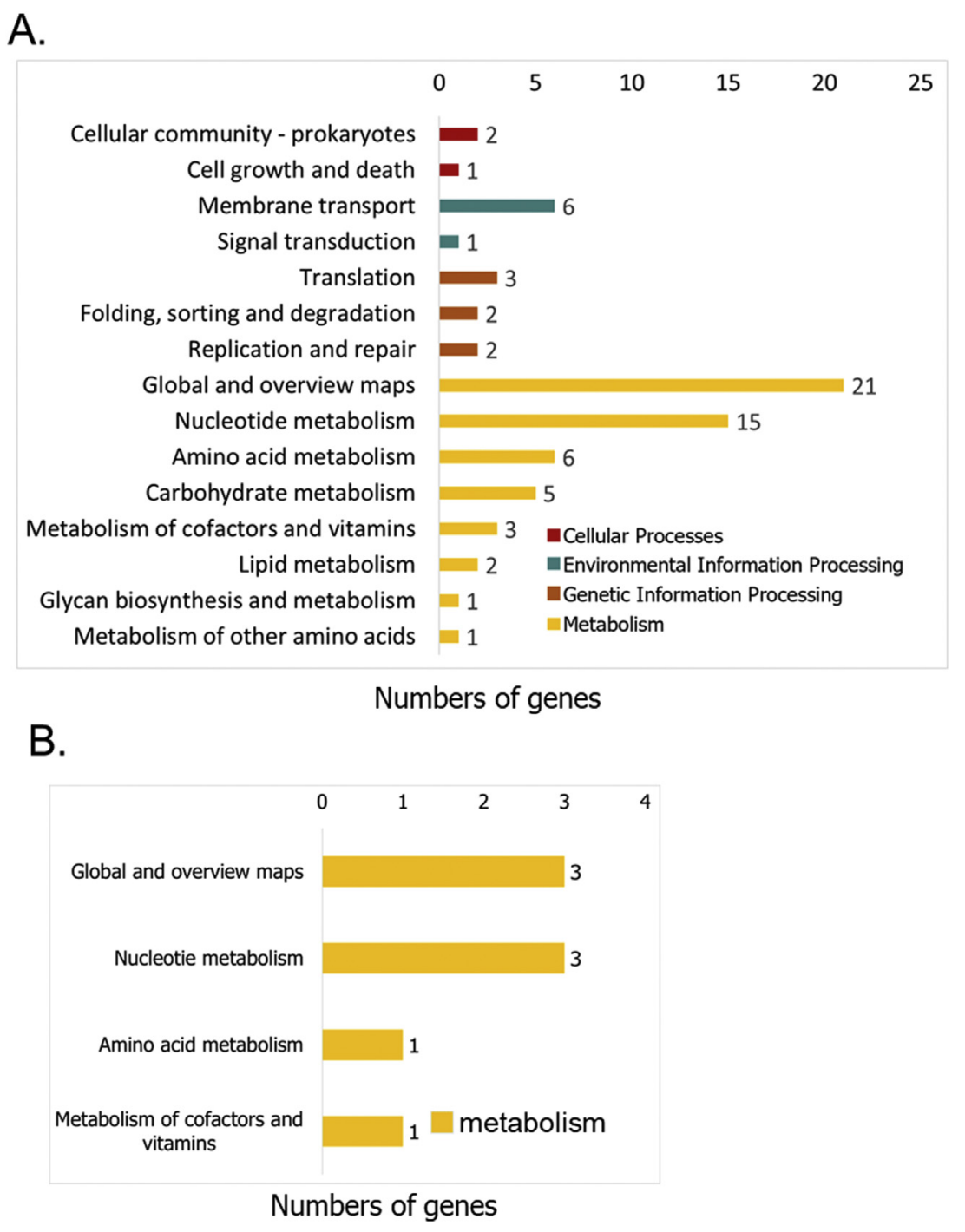

Fig. 2. KEGG classification of lactoferrin treated VS non-treated LGG strain analyzed by using PossionDis method. LGG were cultured at $22{ }^{\circ} \mathrm{C}$ in the presence or absence of BLF incubation from 0 to $72 \mathrm{~h}$. Control $(0 \mathrm{mg} / \mathrm{mL})$ and treated $(1 \mathrm{mg} / \mathrm{mL})$ bacterial samples were analyzed at $60 \mathrm{~h}(\mathrm{~A})$ and $72 \mathrm{~h}$ (B) time interval. The horizontal axis represents the number of genes in that pathway, and vertical axis represents the category of pathway.

metabolism, and galactose metabolism. These upregulated genes of pathways involved in DNA replication, cell wall synthesis, and cell cycle could explain some roles of BLF in promoting the growth of LGG. In contrast, at $72 \mathrm{~h}$ (Fig. 4B), the most enriched pathways in BLF-treated LGG were only down-regulated DEGs of pathways including purine metabolism, biosynthesis of antibiotics, biosynthesis of secondary metabolites, metabolic pathways, one carbon pool by folate, and alanine, aspartate, and glutamate metabolism, and no upregulated DEGs of pathways were identified in LGG at $72 \mathrm{~h}$.

In Fig. 5, GO functional annotation histograms of the DEGs in BLF-treated and control samples are shown. Here, a total of 376 DEGs were clustered into
24 functional groups for the samples harvested at $60 \mathrm{~h}$ (Fig. 5A). Moreover, these DEGs were distributed into 10, 7, and 7 subcategories of biological process, cellular component, and molecular function categories, respectively. Furthermore, the largest subcategories within biological process were "cellular process", "metabolic process", and "membrane part", "cell", and "cell part"; Within the molecular function category, DEGs were distributed within "catalytic activity", and "binding". Moreover, for samples collected at $72 \mathrm{~h}$ (Fig. 5B), only 18 DEGs were clustered into 8 functional groups. These DEGs were distributed into 2, 4, and 2 subcategories of biological process, cellular component, and molecular function categories, respectively. Furthermore, the "cellular process", "metabolic process", 

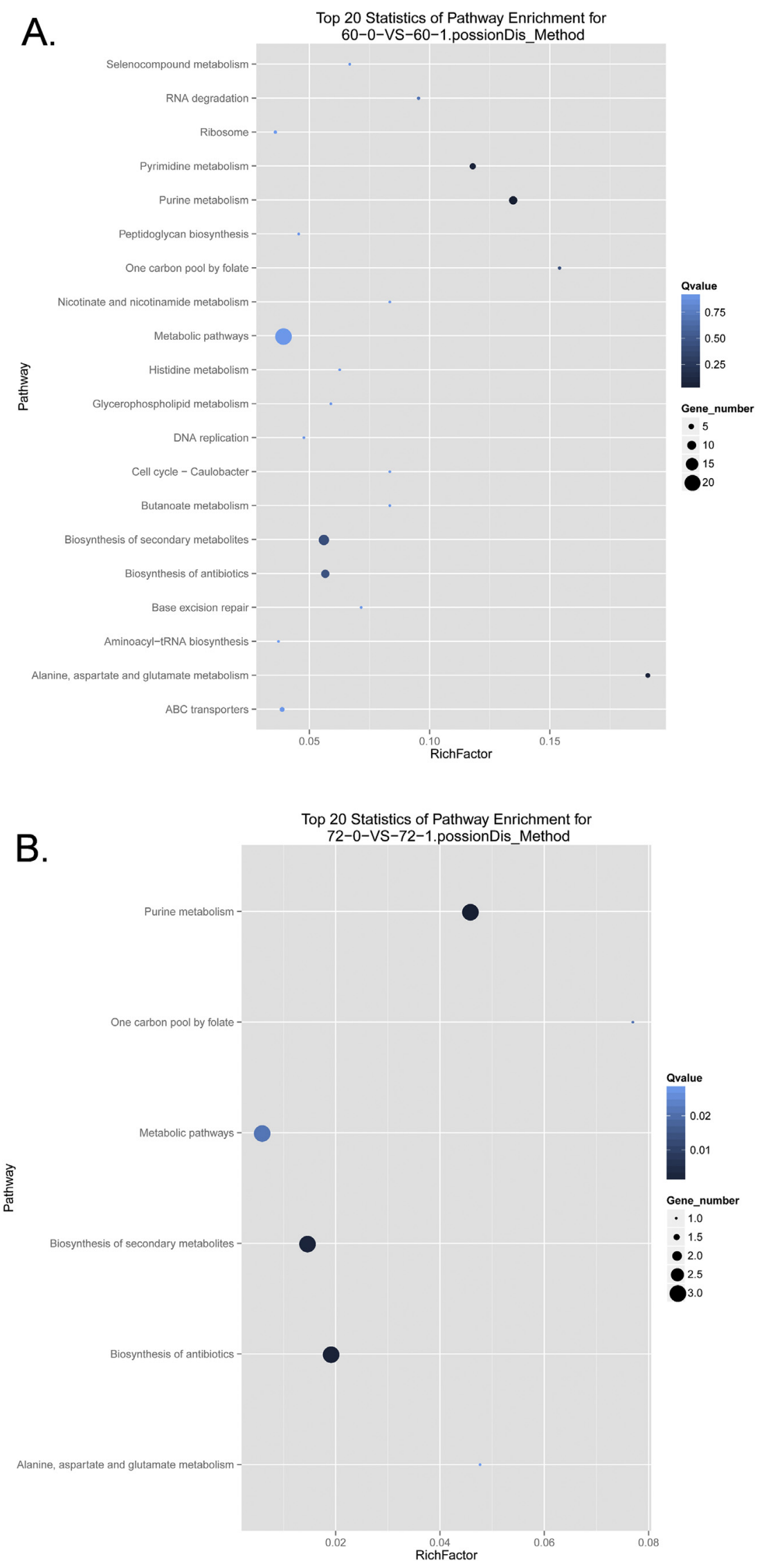

Fig. 3. Scatterplot of the top 20 enriched KEGG pathways of lactoferrin treated VS non-treated LGG. LGG were cultured at $22{ }^{\circ} \mathrm{C}$ in the presence or absence of BLF incubation from 0 to $72 \mathrm{~h}$. Control $(0 \mathrm{mg} / \mathrm{mL})$ and treated (1 mg/mL of BLF) bacterial samples were analyzed at $60 \mathrm{~h}(\mathrm{~A})$ and $72 \mathrm{~h}(\mathrm{~B})$ time interval. The pathway names are represented on the vertical axis, and the horizontal axis represents the pathways corresponding to the rich factor. The ratio of the number of DEGs and all annotated genes in the pathway is defined as the rich factor, and $q \leq 0.05$ as significantly enriched. 

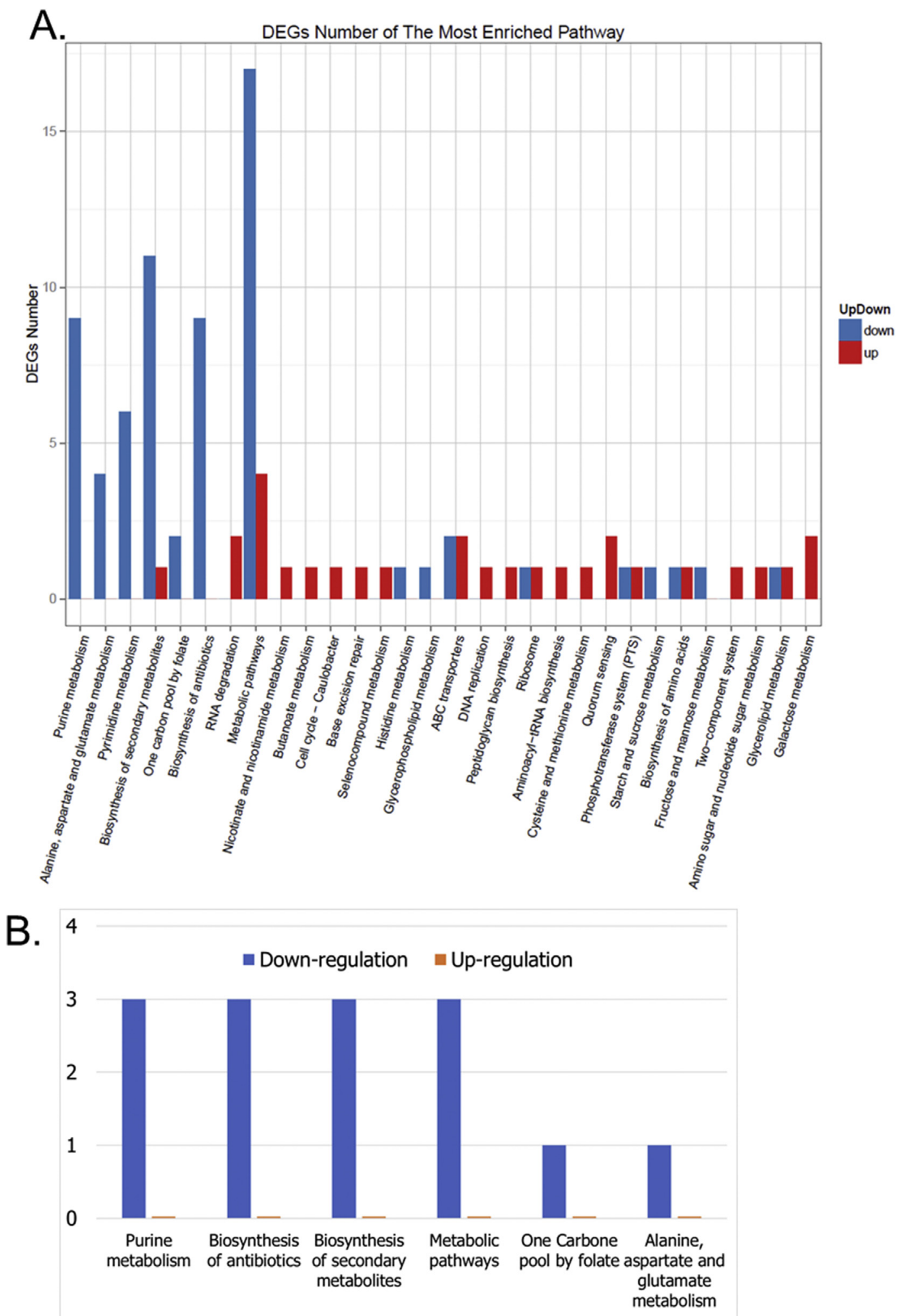

Fig. 4. DEGs numbers of the most enriched pathway of lactoferrin treated VS non-treated LGG strain. LGG were cultured with or without 1-32 mg/mL BLF incubation from 0 to $72 \mathrm{~h}$. Control $(0 \mathrm{mg} / \mathrm{mL})$ and treated $(1 \mathrm{mg} / \mathrm{mL})$ bacterial samples were analyzed at $60 \mathrm{~h}(\mathrm{~A})$ and $72 \mathrm{~h}(\mathrm{~B})$ time interval. The horizontal axis represents the number of genes enriched in that pathway, and these genes of pathways were shown according to the levels of the enriched factors as the strongest enriched DEGs of particular pathway were shown at the left side. 


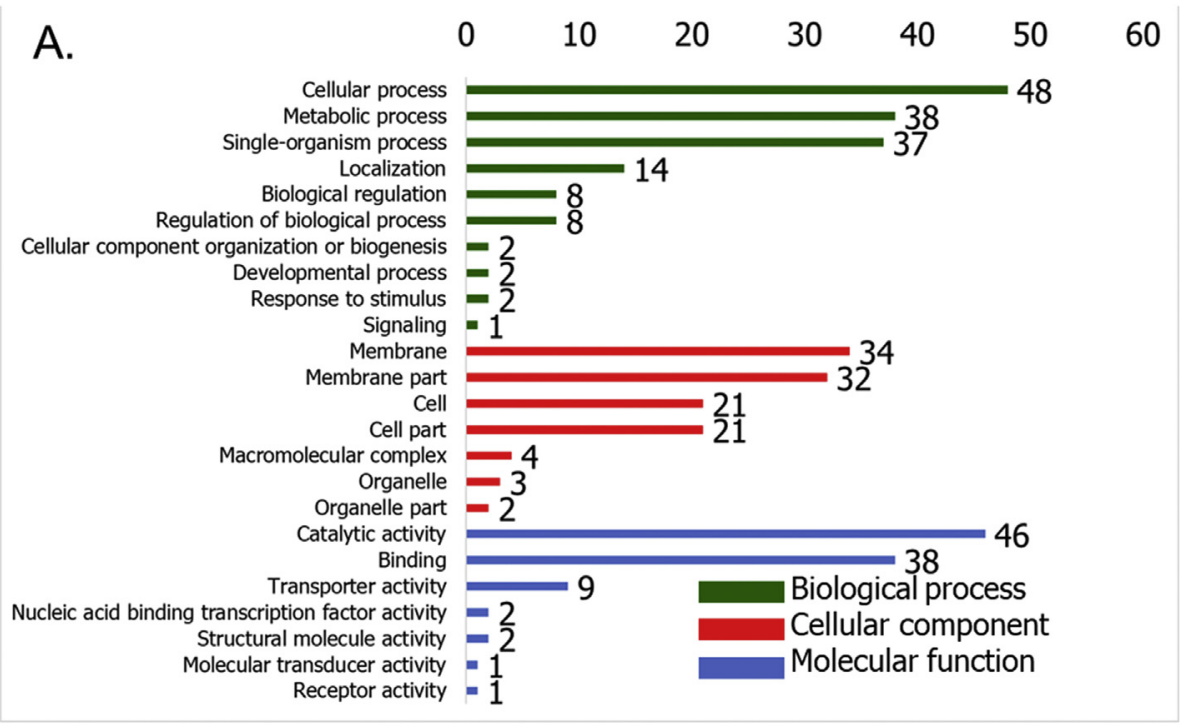

Number of Genes

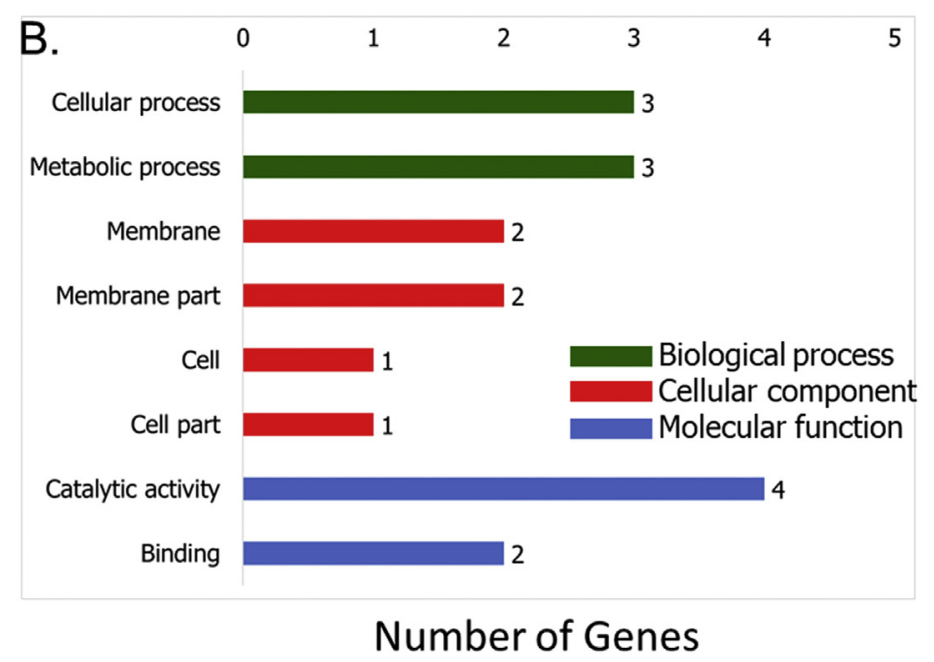

Fig. 5. Functional analysis of GO annotation. The horizontal axis represents the gene number, and the number of genes is considered the difference in the proportion of the total, and the vertical axis represents the three basic GO categories. LGG were cultured with or without 1-32 mg/mL BLF incubation from 0 to $72 \mathrm{~h}$. Control $(0 \mathrm{mg} / \mathrm{mL})$ and treated $(1 \mathrm{mg} / \mathrm{mL})$ bacterial samples were analyzed at $60 \mathrm{~h}$ (A) and $72 \mathrm{~h}$ (B) time interval.

"membrane part", "cell" and "cell part", "catalytic activity", and "binding" subcategories were the most distributed functions.

\section{Discussion}

In our recent reports, BLF supplementation was shown to promote the growth of a series of specific probiotic strains in a dose-dependent manner, as well as promote and re-start the growth of LGG following retardation by a cold stress environment (about $22{ }^{\circ} \mathrm{C}$ ) [6,7]. However, to date, the molecular mechanism of BLF as a prebiotic agent is still unclear. Therefore, in the present study, a global transcriptomic analysis between BLF-treated and untreated LGG cultured at $22{ }^{\circ} \mathrm{C}$ was conducted. Here, we investigated the global gene expression profile at two time points, $60 \mathrm{~h}$ and $72 \mathrm{~h}$. Firstly, at $60 \mathrm{~h}$, the growth of LGG control was still strongly retarded by sub-optimal culturing conditions (cold environment) but BLF supplementation had already boosted the growth of LGG by this time in a dosedependent manner. Second, at $72 \mathrm{~h}$, LGG seemed to have adapted to the cold stress and started to regrow again. However, BLF supplementation still strongly enhanced the growth of LGG at this period 
of time. Notably, the lowest concentration of BLF supplementation tested $(1 \mathrm{mg} / \mathrm{mL})$ was enough to promote the growth of LGG both at $60 \mathrm{~h}$ and $72 \mathrm{~h}$. Therefore, we investigated the transcriptome profiles of LGG treated with $1 \mathrm{mg} / \mathrm{mL}$ BLF between two time intervals. Our data reveals that BLF indeed exerts similar but also different global gene expression profiles in LGG samples harvested at the two time points.

As shown in the present study, the significantly up-regulated core genes in BLF-treated LGG at $60 \mathrm{~h}$ can be classified into the stress- or defense-related responses. For example, expression of genes related to an MFS transporter, a cold-shock protein (CspA family), and a gene encoding LytR family transcriptional regulator and MerR family transcriptional regulator (activator of bmr gene) are strongly enhanced by BLF supplementation. The roles of these genes in prokaryotic bacteria have been previously reported to help different bacterial species to survive or grow under sub-optimal conditions. For example, the MFS transporter has been annotated as a member of the family of multidrug resistance transporters that can act as a defense mechanism against inhibitory compounds by extruding a wide variety of structurally dissimilar substrates from the cytoplasm including antibiotics, bile salts, and peptides [29,30]. In addition, this transporter also displays physiological roles in allowing the bacterium to survive in its ecological niche [31]. In the present study, we also identified expression of another two $\mathrm{ABC}$ transporter-related genes that are promoted by BLF. Thus, BLF supplementation may promote several transporterrelated genes to allow for LGG growth in a cold environment.

As for the BLF-upregulated Csps that we identified, previous findings have suggested that Csps may regulate the expression of cold-induced genes, such as anti-terminators [32], and the expression of CspA in a Lactobacillus casei strain was induced after a temperature downshift from $37{ }^{\circ} \mathrm{C}$ to $20^{\circ} \mathrm{C}$. In addition, a CspA mutant strain was constructed and displayed reduced growth rate compared with the wild type at both optimal and low temperatures, revealing that CspA plays an important role in the physiology of $L$. casei related to cell growth, carbohydrate transport, carbon metabolism, and cold shock [33]. Therefore, CspA may play similar roles in LGG, and the up-regulated CspA could play major roles in driving LGG growth in the cold environment.

Regarding the BLF-upregulated LytR related gene (LytR family transcriptional regulator), the LytRCps-Psr family proteins are commonly present in
Gram-positive bacteria, and these proteins are reported to be required for the surface attachment of both capsular polysaccharides and teichoic acids of pneumococci. Furthermore, the $\Delta$ lytR mutant can impair the growth of Streptococcus pneumoniae, and addition of recombinant LytR protein to the culture medium restores the growth in a dose-dependent manner [34], indicating that LytR also plays physiology roles in maintaining the growth of specific bacterial strains. In support of this, several studies have identified links between growth phase, cell density, and expression of the LytR/CpsA/Psr family in several bacterial strains $[35,36]$. Therefore, in the present study, the enhanced expression of LytR family transcriptional regulator by BLF may boost the function of an integral component of the membrane to help LGG grow in a cold environment.

In this study, BLF also enhanced the expression of $b m r R$, which is a general stress protein that belongs to a group of transcriptional activators (MerR family) of the bmr-bmrR operon. This regulator controls the expression of the Bmr efflux pump in response to a diverse array of cationic antibiotics, dyes, and disinfectants, which are also transported by Bmr and other efflux pumps [37]. Therefore, the roles of MerR family could be similar to the roles of MFS transporters and $A B C$ transporters that are responsible for extruding unfavorable components of bacteria to survive in stress conditions.

At $60 \mathrm{~h}$, BLF supplementation was also upregulated the expression of a gene encoding the cell division protein GpsB by 1.21 fold (Log2 Fold change). It has been shown that GpsB is widely conserved in the Firmicutes phylum and is linked to cell growth and division in Gram-positive bacteria [38-40].

Collectively, previous studies have found that some cold stress-, defense-, and cell division-related genes or products are involved in survival and growth of specific prokaryotic bacterial species. Our data provide new evidence supporting that LGG may also employ these mechanisms to survive in a cold environment, and BLF supplementation could promote these protection effects.

We have explained the roles of BLF on up-regulation of expression of some core genes that can enhance the growth of LGG under a cold environment at $60 \mathrm{~h}$. In support of this, the KEGG pathway and the GO functional analyses have confirmed that BLF supplementation modulated robust genes of pathways that are involved to metabolism, DNA replication, and cell wall synthesis (Fig. 4). However, at $72 \mathrm{~h}$, BLF supplementation seems to have affected another mechanism to boost the growth of LGG. It should be noted that, at $72 \mathrm{~h}$, the LGG control had already adapted to the cold environment and started 
to partially regrow again (Fig. 1). Thus, we believe that some genes or products involved in cold stress responses must have been revoked by LGG control at this stage. Indeed, at this time point, our findings from the PossionDis, KEGG pathway, and GO analyses all support that BLF plays fewer regulating roles on transcriptome gene profiles than that at $60 \mathrm{~h}$ time point. For example, at $72 \mathrm{~h}$, the PossionDis analysis revealed only one gene that was up-regulated and six genes that were down-regulated in BLF-treated LGG. Moreover, for KEGG pathway analysis of the $60 \mathrm{~h}$ time interval, the significantly DEGs were mapped into four categories of pathways: cellular processes (3 genes), environmental information processing (7 genes), genetic information processing ( 7 genes), and metabolism (53 genes).

In contrast, at $72 \mathrm{~h}$, the significantly DEGs were mapped to only one category, the metabolism category (8 genes). In concordance with the fewer pathways modulated by BLF at $72 \mathrm{~h}$ using KEGG pathway analysis, the GO term analysis also revealed that fewer pathways and functions were modulated by BLF supplementation at $72 \mathrm{~h}$. Furthermore, PossionDis approach analysis identified that only seven DEGs were modulated by BLF and no stress- or cell division-related DEGs were affected by BLF incubation. Because LGG control were able to adapt to the cold stress state and restarted to grow again at $72 \mathrm{~h}$, we speculate that the stress-, defense-, or cell division-related genes were revoked in the absence of BLF supplementation at $72 \mathrm{~h}$. Thus, the transcriptome analysis identified fewer molecular pathways and genes that were further modulated in BLF-treated LGG. However, BLF strongly promoted the growth of LGG at both time points studied. Therefore, these DEGs and pathways that were identified to be modulated by BLF at both time points may play core roles to help LGG resist the cold environment.

Our data show that, in BLF-treated LGG at $60 \mathrm{~h}$, although quite a few genes involved in pathways related to metabolism, DNA replication, and cell wall synthesis were promoted by BLF treatment, the most enriched KEGG pathways in BLF-treated LGG were actually down-regulated pathways. These down-regulated pathways include some DEGs related to purine metabolism, alanine, aspartate, and glutamate metabolism, pyrimidine metabolism, biosynthesis of secondary metabolites, one carbon pool by folate, and biosynthesis of antibiotics. Furthermore, in BLF-treated LGG, although fewer numbers of DEGs and pathways were identified and mapped at $72 \mathrm{~h}$, the most enriched KEGG pathways were still down-regulated pathways. These include genes related to purine metabolism, biosynthesis of antibiotics, biosynthesis of secondary metabolites, metabolic pathways, one carbon pool by folate, and alanine, aspartate, and glutamate metabolism. Therefore, when the growth of LGG control cells were still blocked by the cold environment (at $60 \mathrm{~h}$ ) or when the LGG had adapted to the cold environment and started to regrow again (at $72 \mathrm{~h}$ ), BLF supplementation contributed to down-regulation of the above nucleotide-, specific amino acid-, and carbon pool-related pathways. Overall, we believe these down-regulated metabolic pathways, which are involved in purine, amino acid, pyrimidine, onecarbon metabolism, and secondary metabolites may reduce energy requirements and maintain carbon metabolism balance to help BLF-treated LGG to survive and grow in cold stress conditions. To support our findings, previous transcriptome analyses in L. acetotolerans F28, Lactobacillus plantarum, and beer-spoilage $L$. acetotolerans have suggested that reducing "amino acid transport and metabolism" pathways might be a necessary strategy to maintain carbon and nitrogen metabolism balance to survive under ethanol stress or viable putative non-culturable state $[13,41,42]$.

In conclusion, LGG is one of the most widely investigated and well-documented probiotic bacterial strains, and it has been applied to various fields. LF is a natural protein of mammals that displays extensive physiological functions. We demonstrated previously that LGG could resist the antibacterial activities of BLF, and BLF supplementation could further boost the cold tolerance of LGG. However, it was still unclear about the molecular mechanism of BLF on promoting the growth of LGG. In the present study, though transcriptome analysis, BLF supplementation has been shown to modulate a number of genes that are involved in many central metabolic pathways. In Fig. 6 , the main molecular roles of BLF in promoting the growth of LGG are summarized according to the findings from current study. BLF supplementation could elevate higher expression levels of specific stress, defense, cell division, and transporter response genes that have been reported to be relevant to growth and survival in several bacterial species. In addition, BLF supplementation could also reduce a series of metabolic pathways involved in purine, amino acid, pyrimidine, one-carbon metabolism, and secondary metabolites, and these may help LGG to reduce energy requirements and maintain carbon metabolism balance in order to survive and grow in a cold environment. The combination of the above effects and molecular pathways may render the ability of BLF to strongly boost the cold tolerance of LGG. 


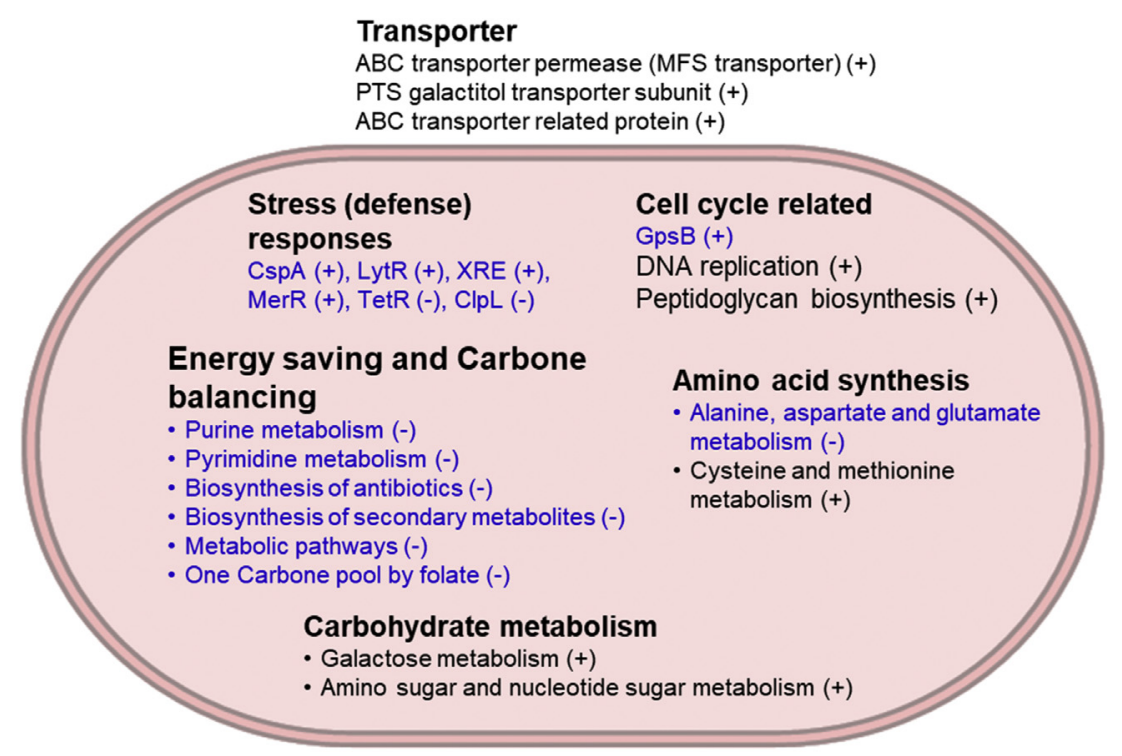

Fig. 6. The central molecular pathways or genes which are modulated by BLF supplementation in LGG under a cold environment incubation. Increased and decreased expression is indicated by plus symbol ' + ' and minus symbol '-' respectively.

However, further experiments are needed to confirm the adaptive behavior of LGG exerted by BLF by using different experiment design or approaches, and to elucidate the adaptive behavior of LGG exerted by BLF could help to both elucidate the prebiotic activity of BLF and understand the cold tolerance mechanism of LGG. For example, the identified DEGs in KEGG term-enriched terms may help to secure core candidate genes for further functional studies on the molecular mechanisms of stress tolerance in LGG. BLF could be potentially applied in future to promote or maintain the growth of specific probiotics cultures under sub-optimal conditions. However, as described elsewhere in this study, the prebiotic ability of BLF is actually bacterial strain-dependent, and thus, BLF may modulate different DEGs and putative molecular regulatory networks in different probiotic strains.

\section{Conflict of interest}

No conflict of interest exists.

\section{Acknowledgements}

This work was supported by the Ministry of Science and Technology under two grants [MOST 1072221-E-131-001-MY2 and MOST 108-2320-B-005006]. 


\section{Appendix A. Supplementary material}
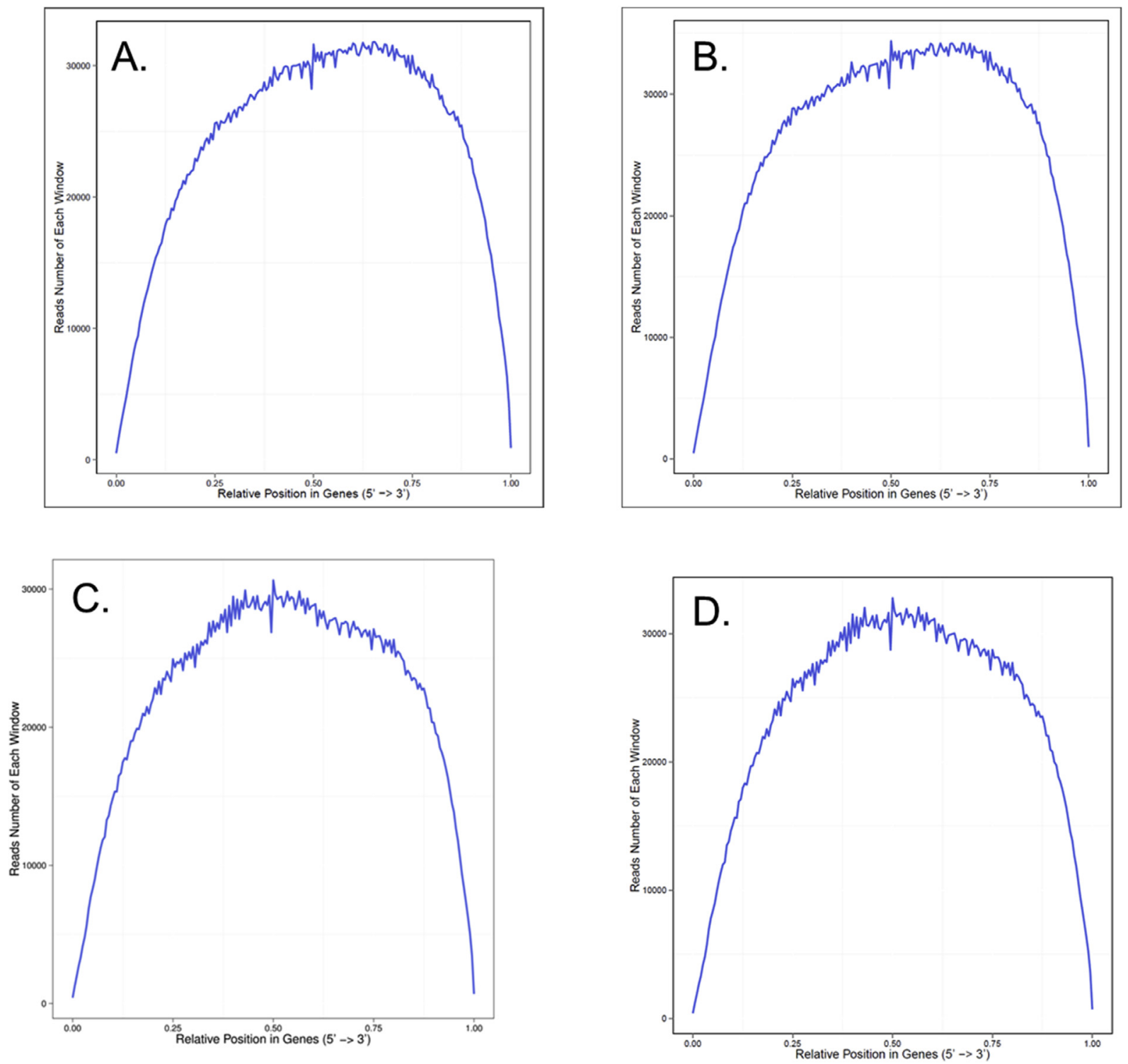

Fig. 1S. Reads distribute on references gene. LGG were cultured at $22{ }^{\circ} \mathrm{C}$ with or without BLF incubation from 0 to $72 \mathrm{~h}$. Gene expression levels of control $(0 \mathrm{mg} / \mathrm{mL})$ and BLF-treated $(1 \mathrm{mg} / \mathrm{mL})$ bacterial samples were analyzed using RNA-seq analysis at $60 \mathrm{~h}$ and $72 \mathrm{~h}$ time interval. (A) samples of $60 \mathrm{~h}$ (control); (B) samples of $60 \mathrm{~h}$ (BLF treated one); (C) samples of $72 \mathrm{~h}$ (control); (D) samples of $72 \mathrm{~h}$ BLF treated one. 

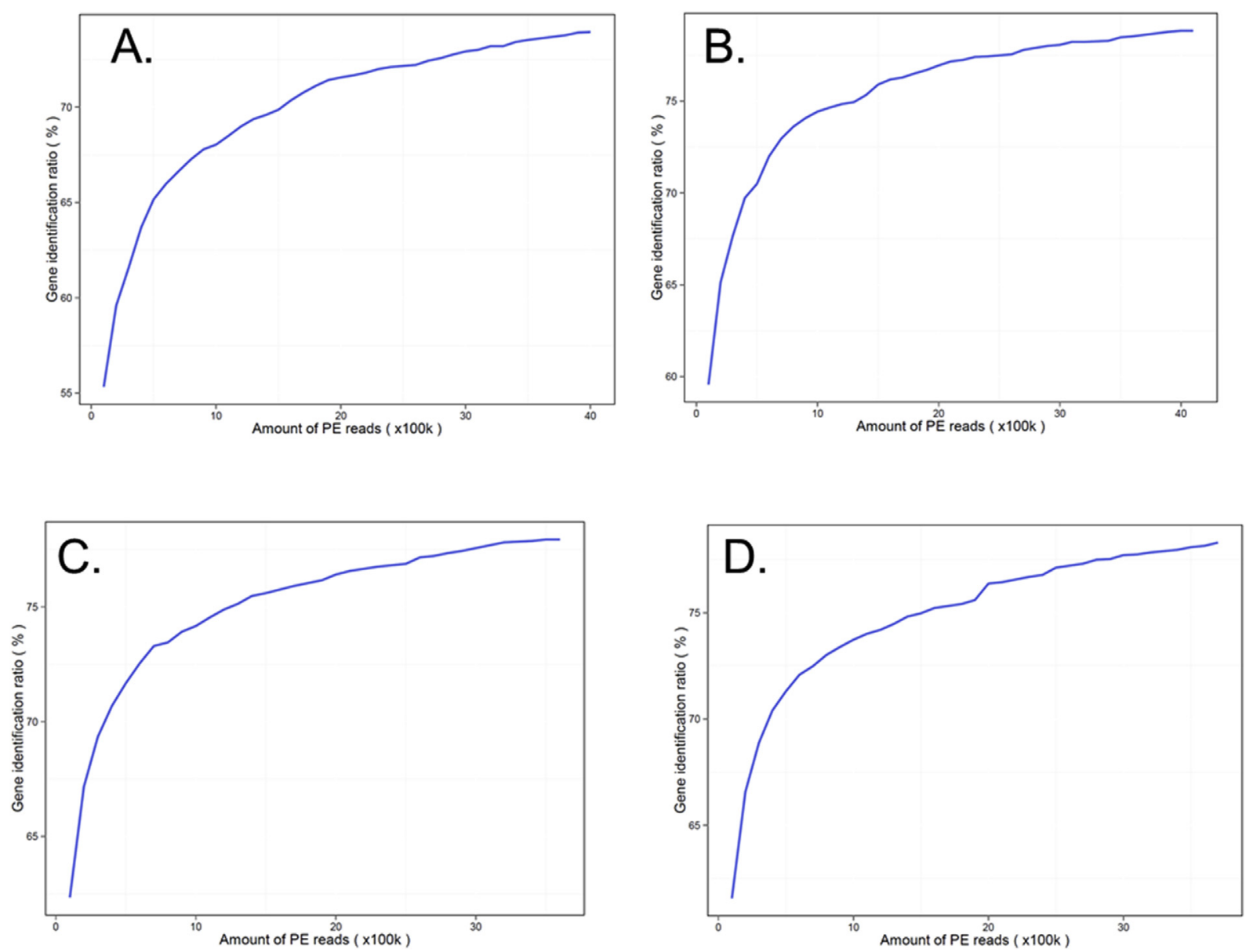

Fig. 2S. Curve of sequencing saturation.X-axis shows the number of clean reads, units is 100 k-extreme value is currently the volume of sequencing. $Y$-axis shows the ratio of identified gene number to number of total gene reported in database. (A) samples of $60 \mathrm{~h}$ (control); (B) samples of $60 \mathrm{~h}$ (BLF treated one); (C) samples of $72 \mathrm{~h}$ (control); (D) samples of $72 \mathrm{~h}$ BLF treated one.

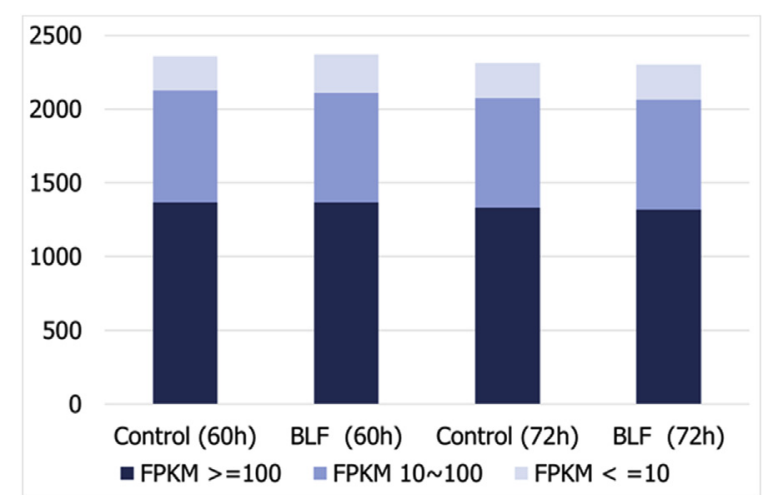

Fig. 3S. Gene expression in each sample. Measurement of the abundance of expression for each assembled transcript was done using the Fragments per Kilobase of exon model per Million mapped reads (FPKM) values. 

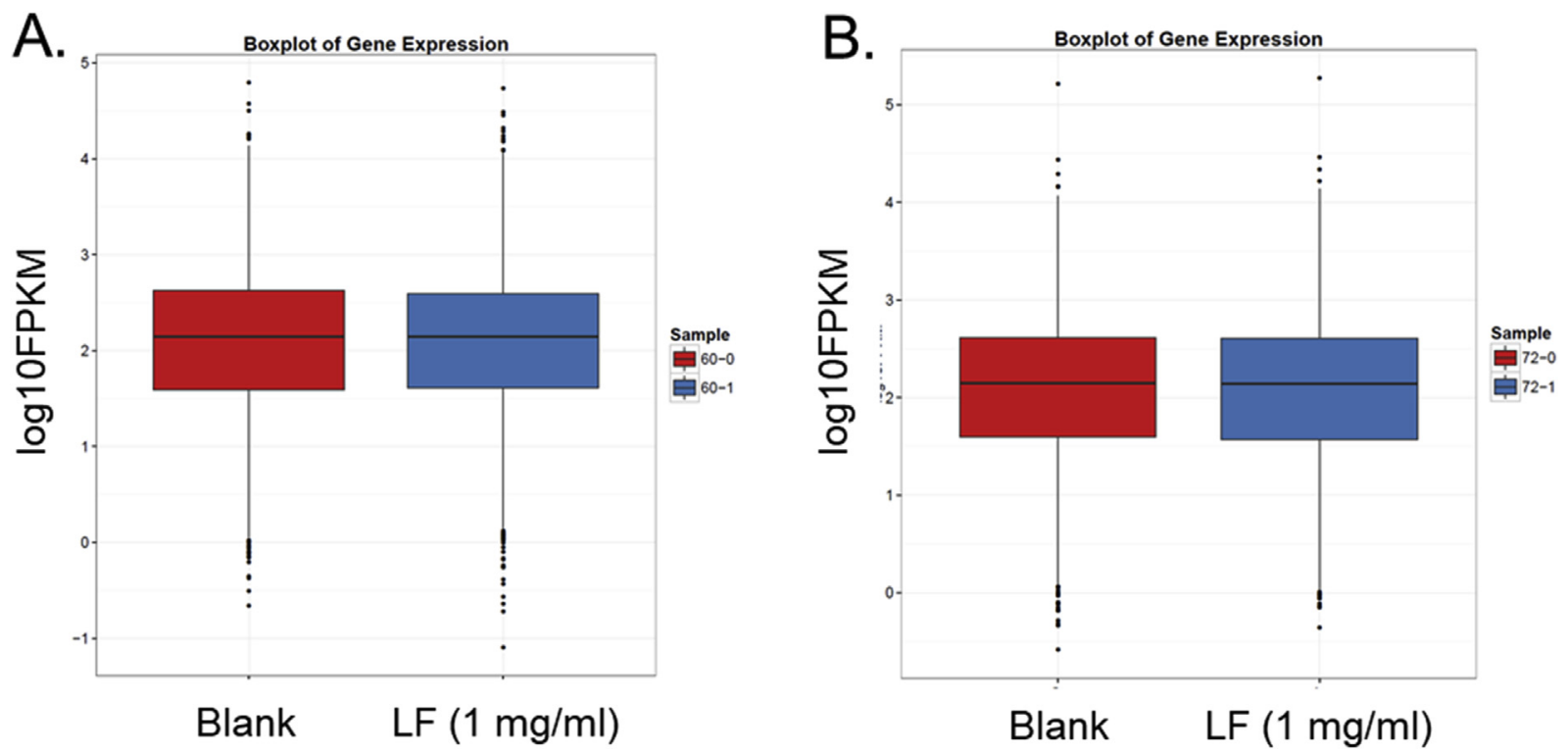

Fig. 4S. Boxplot of gene expression. Gene expression in each samples. LGG were cultured at $22{ }^{\circ} \mathrm{C}$ with or without BLF incubation from 0 to $72 \mathrm{~h}$. Gene expression levels of control $(0 \mathrm{mg} / \mathrm{mL})$ and BLF-treated $(1 \mathrm{mg} / \mathrm{mL})$ bacterial samples were analyzed using $R N A$-seq analysis at $60 \mathrm{~h}(A)$ and $72 \mathrm{~h}$ (B) time interval. Measurement of the abundance of expression for each assembled transcript was done using the Fragments per Kilobase of exon model per Million mapped reads (FPKM) values. The box depicts the interquartile range between the first and third quartiles (25 th and 75 th percentiles, respectively) and the line inside the box indicates the median.

A.

Hierarchical Clustering of Exp(inter:2321 Genes)

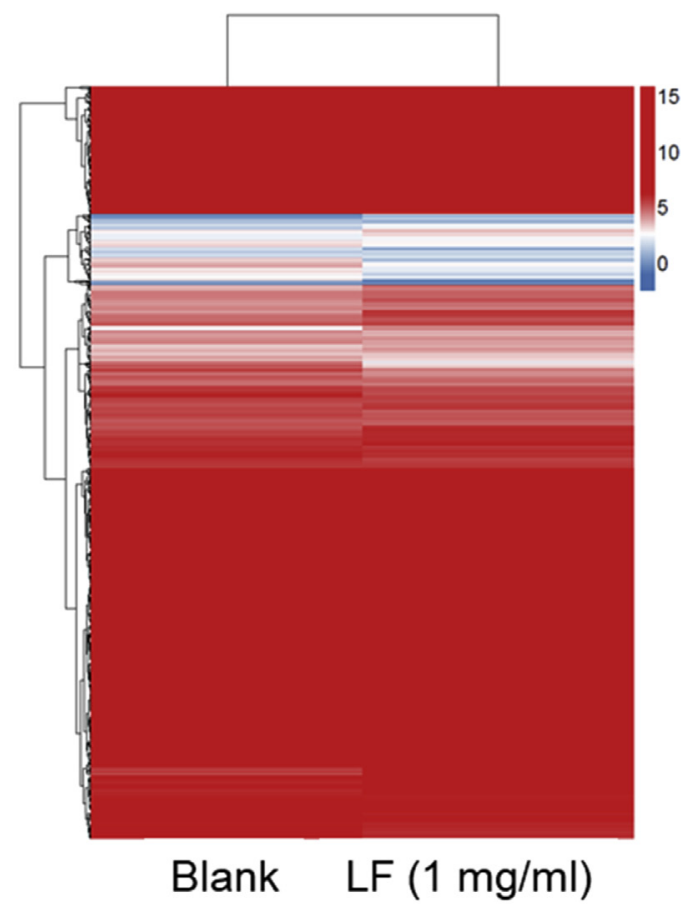

B.
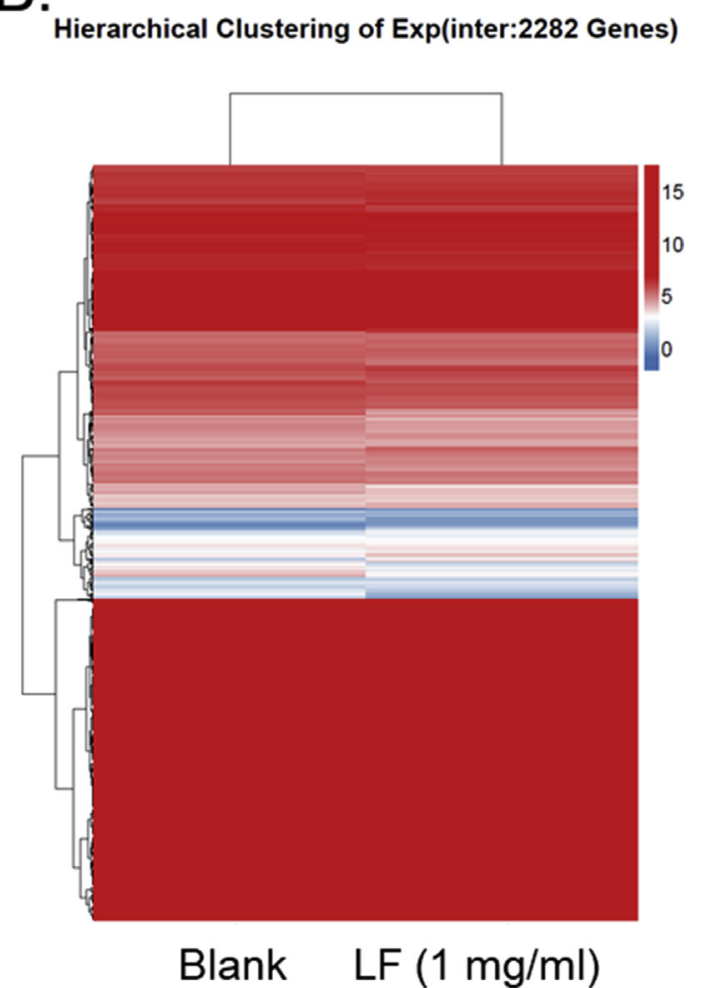

Fig. 5S. Hierarchical clustering of interactively expression genes LGG were cultured at $22{ }^{\circ} \mathrm{C}$ in the presence of absence of BLF incubation from 0 to 72 $\mathrm{h}$. Gene expression levels of control $(0 \mathrm{mg} / \mathrm{mL})$ and BLF-treated $(1 \mathrm{mg} / \mathrm{mL})$ bacterial samples were analysed using $R N A$-seq analysis at $60 \mathrm{~h}(\mathrm{~A}) 72 \mathrm{~h}$ (B) time interval. 


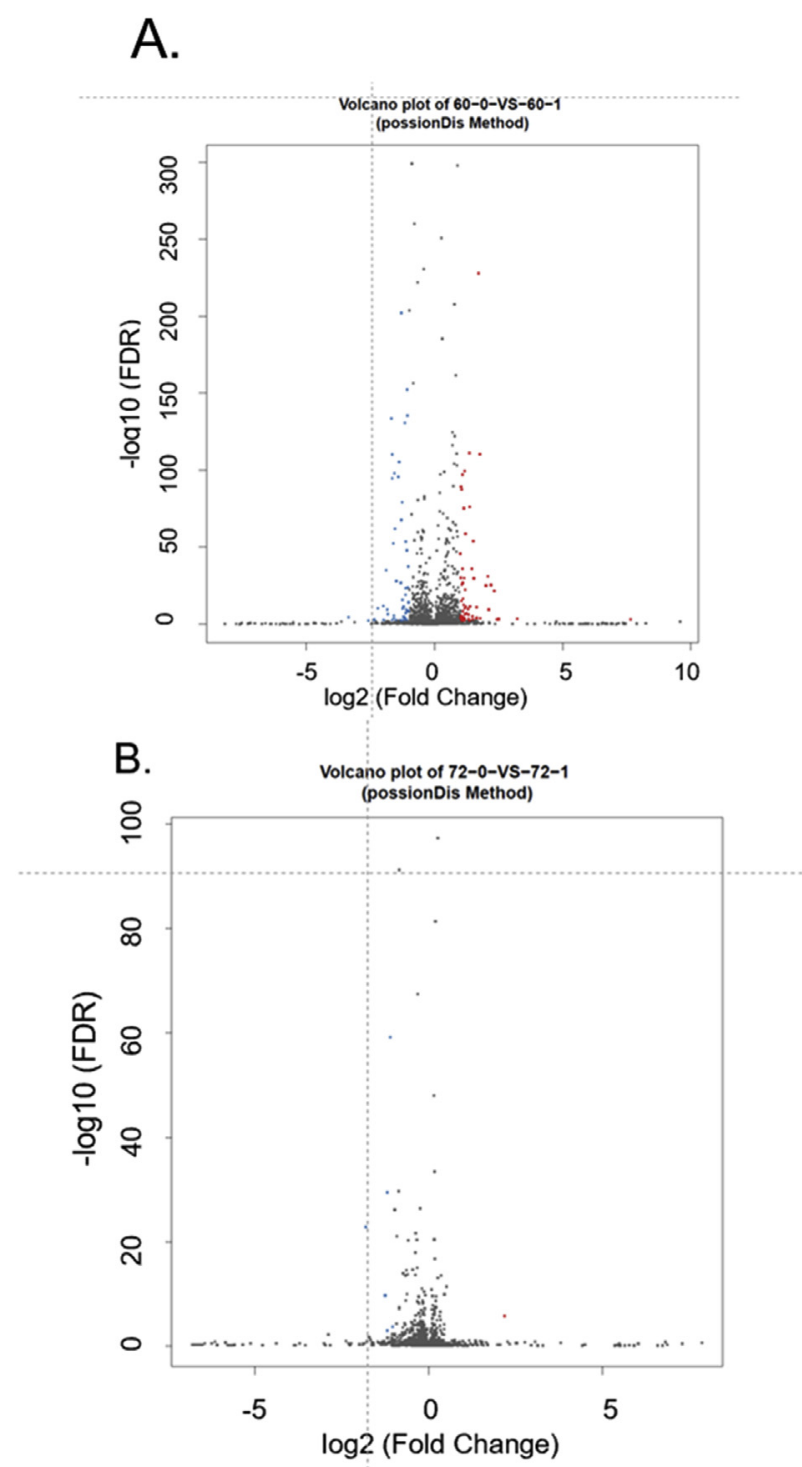

Fig. 6S. Volcano plot of different expression genes (DEGs) between samples treated with or without BLF analyzed using possionDis method. LGG were cultured at $22{ }^{\circ} \mathrm{C}$ with or without BLF incubation from 0 to $72 \mathrm{~h}$. Gene expression levels of control $(0 \mathrm{mg} / \mathrm{mL})$ and BLF-treated (1 mg/mL) bacterial samples were analyzed using RNA-seq analysis at $60 \mathrm{~h}(\mathrm{~A})$ and $72 \mathrm{~h}$ (B) time interval; Up-regulated significantly DEGs were shown in red (Log2FoldChange $\geq 1$ or FDR $\leq 0.005$ ), down-regulated significantly DEGs were shown in blue (Log2FoldChange $\leq-1$ or FDR $\leq 0.005)$, and nonregulated significantly DEGs were shown in grey (Log2FoldChange $<1$ or FDR $>0.005$ ).

\section{References}

[1] Valenti P, Antonini G. Lactoferrin: an important host defence against microbial and viral attack. Cell Mol Life Sci 2005;62: 2576-87.

[2] Frioni A, Conte MP, Cutone A, Longhi C, Musci G, di Patti MC, et al. Lactoferrin differently modulates the inflammatory response in epithelial models mimicking human inflammatory and infectious diseases. Biometals 2014;27: 843-56.

[3] Legrand D. Overview of lactoferrin as a natural immune modulator. J Pediatr 2016;173(Suppl):S10-5.
[4] Superti F, De Seta F. Warding off recurrent yeast and bacterial vaginal infections: lactoferrin and lactobacilli. Microorganisms 2020;8.

[5] Chen PW, Jheng TT, Shyu CL, Mao FC. Antimicrobial potential for the combination of bovine lactoferrin or its hydrolysate with lactoferrin-resistant probiotics against foodborne pathogens. J Dairy Sci 2013;96:1438-46.

[6] Chen PW, Ku YW, Chu FY. Influence of bovine lactoferrin on the growth of selected probiotic bacteria under aerobic conditions. Biometals 2014;27:905-14.

[7] Chen PW, Liu ZS, Kuo TC, Hsieh MC, Li ZW. Prebiotic effects of bovine lactoferrin on specific probiotic bacteria. Biometals 2017;30:237-48. 
[8] Chen PW, Jheng TT, Shyu CL, Mao FC. Synergistic antibacterial efficacies of the combination of bovine lactoferrin or its hydrolysate with probiotic secretion in curbing the growth of meticillin-resistant Staphylococcus aureus. J Med Microbiol 2013;62:1845-51.

[9] Wang Z, Gerstein M, Snyder M. RNA-Seq: a revolutionary tool for transcriptomics. Nat Rev Genet 2009;10:57-63.

[10] Marioni JC, Mason CE, Mane SM, Stephens M, Gilad Y. RNA-seq: an assessment of technical reproducibility and comparison with gene expression arrays. Genome Res 2008; 18:1509-17.

[11] Lawley B, Sims IM, Tannock GW. Whole-transcriptome shotgun sequencing (RNA-seq) screen reveals upregulation of cellobiose and motility operons of Lactobacillus ruminis L5 during growth on tetrasaccharides derived from barley beta-glucan. Appl Environ Microbiol 2013;79:5661-9.

[12] Laakso K, Koskenniemi K, Koponen J, Kankainen M, Surakka A, Salusjarvi T, et al. Growth phase-associated changes in the proteome and transcriptome of Lactobacillus rhamnosus GG in industrial-type whey medium. Microb Biotechnol 2011;4:746-66.

[13] Yang X, Teng K, Zhang J, Wang F, Zhang T, Ai G, et al. Transcriptome responses of Lactobacillus acetotolerans F28 to a short and long term ethanol stress. Sci Rep 2017; $7: 2650$.

[14] Wong SS, Quan Toh Z, Dunne EM, Mulholland EK, Tang ML, Robins-Browne RM, et al. Inhibition of Streptococcus pneumoniae adherence to human epithelial cells in vitro by the probiotic Lactobacillus rhamnosus GG. BMC Res Notes 2013;6:135.

[15] Hudault S, Lievin V, Bernet-Camard MF, Servin AL. Antagonistic activity exerted in vitro and in vivo by Lactobacillus casei (strain GG) against Salmonella typhimurium C5 infection. Appl Environ Microbiol 1997;63:513-8.

[16] Zhou B, Zhang D. Antibacterial effects of bacteriocins isolated from Lactobacillus rhamnosus (ATCC 53103) in a rabbit model of knee implant infection. Exp Ther Med 2018;15: 2985-9.

[17] Meurman J, Antila H, Korhonen A, SJEjoos Salminen. Effect of Lactobacillus rhamnosus strain GG (ATCC 53103) on the growth of Streptococcus sobrinus in vitro. Euro J Oral Sci 1995; 103:253-8.

[18] Miettinen M, Matikainen S, Vuopio-Varkila J, Pirhonen J, Varkila K, Kurimoto M, et al. Lactobacilli and streptococci induce interleukin-12 (IL-12), IL-18, and gamma interferon production in human peripheral blood mononuclear cells. Infect Immun 1998;66:6058-62.

[19] Veckman V, Miettinen M, Pirhonen J, Siren J, Matikainen S, Julkunen I. Streptococcus pyogenes and Lactobacillus rhamnosus differentially induce maturation and production of Th1-type cytokines and chemokines in human monocytederived dendritic cells. J Leukoc Biol 2004;75:764-71.

[20] Fang H, Elina T, Heikki A, Seppo S. Modulation of humoral immune response through probiotic intake. FEMS Immunol Med Microbiol 2000;29:47-52.

[21] Mekkes MC, Weenen TC, Brummer RJ, Claassen E. The development of probiotic treatment in obesity: a review. Benef Microbes 2014;5:19-28.

[22] Hibberd PL, Kleimola L, Fiorino A-M, Botelho C, Haverkamp M, Andreyeva I, et al. No evidence of harms of probiotic Lactobacillus rhamnosus GG ATCC 53103 in healthy elderly - a phase I open label study to assess safety, tolerability and cytokine responses, vol. 9; 2014.

[23] Salminen SJ, Donohue DJNT. Safety assessment of Lactobacillus strain GG (ATCC 53103), vol. 31; 1996. p. 16S.
[24] Kim D, Langmead B, Salzberg SL. HISAT: a fast spliced aligner with low memory requirements. Nat Method 2015;12: 357-60.

[25] Audic S, Claverie JM. The significance of digital gene expression profiles. Genome Res 1997;7:986-95.

[26] Kanehisa M, Araki M, Goto S, Hattori M, Hirakawa M, Itoh $\mathrm{M}$, et al. KEGG for linking genomes to life and the environment. Nucleic Acids Res 2008;36:D480-4.

[27] de Hoon MJ, Imoto S, Nolan J, Miyano S. Open source clustering software. Bioinformatics 2004;20:1453-4.

[28] Eisen MB, Spellman PT, Brown PO, Botstein D. Cluster analysis and display of genome-wide expression patterns. Proc Natl Acad Sci U S A 1998;95:14863-8.

[29] Putman M, van Veen HW, Konings WN. Molecular properties of bacterial multidrug transporters. Microbiol Mol Biol Rev 2000;64:672-93.

[30] Pfeiler EA, Klaenhammer TR. Role of transporter proteins in bile tolerance of Lactobacillus acidophilus. Appl Environ Microbiol 2009;75:6013-6.

[31] Piddock LJ. Multidrug-resistance efflux pumps - not just for resistance. Nat Rev Microbiol 2006;4:629-36.

[32] Bae W, Xia B, Inouye M, Severinov K. Escherichia coli CspAfamily RNA chaperones are transcription antiterminators. Proc Natl Acad Sci U S A 2000;97:7784-9.

[33] Sauvageot N, Beaufils S, Maze A, Deutscher J, Hartke A. Cloning and characterization of a gene encoding a coldshock protein in Lactobacillus casei. FEMS Microbiol Lett 2006;254:55-62.

[34] Ye W, Zhang J, Shu Z, Yin Y, Zhang X, Wu K. Pneumococcal LytR protein is required for the surface attachment of both capsular polysaccharide and teichoic acids: essential for pneumococcal virulence. Front Microbiol 2018;9:1199.

[35] Chatfield $\mathrm{CH}$, Koo H, Quivey RG. The putative autolysin regulator LytR in Streptococcus mutans plays a role in cell division and is growth-phase regulated. Microbiology 2005; 151:625-31.

[36] Rossi J, Bischoff M, Wada A, Berger-Bachi B. MsrR, a putative cell envelope-associated element involved in Staphylococcus aureus sarA attenuation. Antimicrob Agents Chemother 2003;47:2558-64.

[37] Ahmed M, Borsch CM, Taylor SS, Vazquez-Laslop N, Neyfakh AA. A protein that activates expression of a multidrug efflux transporter upon binding the transporter substrates. J Biol Chem 1994;269:28506-13.

[38] Fleurie A, Manuse S, Zhao C, Campo N, Cluzel C, Lavergne JP, et al. Interplay of the serine/threonine-kinase StkP and the paralogs DivIVA and GpsB in pneumococcal cell elongation and division. PLoS Genet 2014;10:e1004275.

[39] Cleverley RM, Rutter ZJ, Rismondo J, Corona F, Tsui HT, Alatawi FA, et al. The cell cycle regulator GpsB functions as cytosolic adaptor for multiple cell wall enzymes. Nat Commun 2019;10:261.

[40] Eswara PJ, Brzozowski RS, Viola MG, Graham G, Spanoudis C, Trebino C, et al. An essential Staphylococcus aureus cell division protein directly regulates FtsZ dynamics. Elife 2018;7.

[41] Liu J, Deng Y, Peters BM, Li L, Li B, Chen L, et al. Transcriptomic analysis on the formation of the viable putative non-culturable state of beer-spoilage Lactobacillus acetotolerans. Sci Rep 2016;6:36753.

[42] van Bokhorst-van de Veen $H$, Abee $T$, Tempelaars $M$, Bron PA, Kleerebezem M, Marco ML. Short- and long-term adaptation to ethanol stress and its cross-protective consequences in Lactobacillus plantarum. Appl Environ Microbiol 2011;77:5247-56. 